\title{
Positivity bounds on double parton distributions
}

\section{Markus Diehl and Tomas Kasemets}

Theory Group, Deutsches Elektronen-Synchroton DESY, Notkestr. 85, 22607 Hamburg, Germany

E-mail: markus.diehl@desy.de, tomas.kasemets@desy.de

Abstract: Double hard scattering in proton-proton collisions is described in terms of double parton distributions. We derive bounds on these distributions that follow from their interpretation as probability densities, taking into account all possible spin correlations between two partons in an unpolarized proton. These bounds constrain the size of the polarized distributions and can for instance be used to set upper limits on the effects of spin correlations in double hard scattering. We investigate the stability of the bounds under leading-order DGLAP evolution to higher scales.

Keywords: QCD Phenomenology, Hadronic Colliders

ARXiv EPRINT: 1303.0842 


\section{Contents}

1 Introduction 1

2 Double parton distributions 2

3 Two-parton spin density matrices 4

4 Positivity bounds $\quad 6$

5 Stability under evolution $\quad 8$

$\begin{array}{lll}5.1 & \text { Evolution of double parton distributions } & 8\end{array}$

5.2 Linear combinations of DPDs 9

$\begin{array}{ll}5.3 & \text { Evolution of the linear combinations } \\ \end{array}$

6 Conclusions 12

$\begin{array}{ll}\text { A Evolution equations and splitting functions } & 12\end{array}$

$\begin{array}{ll}\text { B Elements of a stability proof } & 13\end{array}$

\section{Introduction}

In a time when the dynamics of the strong interaction in hadron-hadron collisions is moving towards the domain of precision physics, there are still aspects that are under poor theoretical and experimental control. One of these aspects is double parton scattering, where two partons from each proton have a hard interaction in a single proton-proton collision. Correlations between the two hard interactions have been the subject of several recent studies [1-8]. The relevance of spin correlations in double parton scattering was pointed out long ago [9, 10] and recently followed up in [11, 12]. The studies in [13] and [14] have shown that spin correlations in the production of two vector bosons by double hard scattering have observable effects both on the interaction rate and on kinematic distributions. Spin correlations between the two partons are quantified by polarized double parton distributions (DPDs), which describe for instance the difference of the probability densities for finding two quarks with equal or with opposite helicities. It was argued in [12] that such correlations need not be small, and a recent study in the MIT bag model [15] indeed found large spin correlations between quarks in the valence region. However, our knowledge of polarized DPDs is still poor at best, and any information about them is of value.

In the present work, we derive model independent constraints on DPDs that follow from their interpretation as probability densities for finding two partons in a specified polarization state. Similar positivity bounds have been derived for single-parton distributions in the form of the Soffer bound [16] and of inequalities for transverse-momentum dependent distributions [17] and generalized parton distributions [18]. 
The structure of this paper is as follows. In the next section we set the stage by introducing the DPDs for different polarizations and parton species. In section 3 we derive the spin density matrices for two partons inside an unpolarized proton, and in section 4 we use these matrices to derive bounds on polarized DPDs. In section 5 we show that the homogeneous leading-order evolution equations preserve these bounds when going to higher scales. We conclude in section 6 and give some technical details in two appendices.

\section{Double parton distributions}

Double parton distributions for quarks and antiquarks have been extensively studied in [12], and we only review the properties important for our purpose. Since we will need a probability interpretation, we restrict ourselves to distributions that are integrated over the transverse parton momenta and that have a trivial color structure. In the parlance of [12] these are collinear color-singlet distributions.

Collinear DPDs depend on the longitudinal momentum fractions $x_{1}$ and $x_{2}$ of the two partons and on the transverse distance $\boldsymbol{y}$ between them. For two partons $a_{1}$ and $a_{2}$ in an unpolarized right-moving proton we write

$$
\begin{aligned}
F_{a_{1} a_{2}}\left(x_{1}, x_{2}, \boldsymbol{y}\right)= & 2 p^{+}\left(x_{1} p^{+}\right)^{-n_{1}}\left(x_{2} p^{+}\right)^{-n_{2}} \int \frac{d z_{1}^{-}}{2 \pi} \frac{d z_{2}^{-}}{2 \pi} d y^{-} e^{i\left(x_{1} z_{1}^{-}+x_{2} z_{2}^{-}\right) p^{+}} \\
& \times\left\langle p\left|\mathcal{O}_{a_{2}}\left(0, z_{2}\right) \mathcal{O}_{a_{1}}\left(y, z_{1}\right)\right| p\right\rangle
\end{aligned}
$$

where $n_{i}=1$ if parton number $i$ is a gluon and $n_{i}=0$ otherwise. We use light-cone coordinates $v^{ \pm}=\left(v^{0} \pm v^{3}\right) / \sqrt{2}$ and the transverse component $\boldsymbol{v}=\left(v^{1}, v^{2}\right)$ for any four-vector $v$. The operators for quarks read

$$
\mathcal{O}_{a_{i}}\left(y, z_{i}\right)=\left.\bar{q}_{i}\left(y-\frac{1}{2} z_{i}\right) \Gamma_{a_{i}} q_{i}\left(y+\frac{1}{2} z_{i}\right)\right|_{z_{i}^{+}=y^{+}=0, \boldsymbol{z}_{i}=\mathbf{0}}
$$

with projections

$$
\Gamma_{q}=\frac{1}{2} \gamma^{+}, \quad \Gamma_{\Delta q}=\frac{1}{2} \gamma^{+} \gamma_{5}, \quad \Gamma_{\delta q}^{j}=\frac{1}{2} i \sigma^{j+} \gamma_{5} \quad(j=1,2)
$$

onto unpolarized quarks $(q)$, longitudinally polarized quarks $(\Delta q)$ and transversely polarized quarks $(\delta q)$. The field with argument $y+\frac{1}{2} z_{i}$ in $\mathcal{O}_{a_{i}}\left(y, z_{i}\right)$ is associated with a quark in the amplitude of a double scattering process and the field with argument $y-\frac{1}{2} z_{i}$ with a quark in the complex conjugate amplitude. The operators for gluons are

$$
\mathcal{O}_{a_{i}}\left(y, z_{i}\right)=\left.\Pi_{a_{i}}^{j j^{\prime}} G^{+j^{\prime}}\left(y-\frac{1}{2} z_{i}\right) G^{+j}\left(y+\frac{1}{2} z_{i}\right)\right|_{z_{i}^{+}=y^{+}=0, \boldsymbol{z}_{i}=\mathbf{0}}
$$

with projections

$$
\Pi_{g}^{j j^{\prime}}=\delta^{j j^{\prime}}, \quad \quad \Pi_{\Delta g}^{j j^{\prime}}=i \epsilon^{j j^{\prime}}, \quad\left[\Pi_{\delta g}^{k k^{\prime}}\right]^{j j^{\prime}}=\tau^{j j^{\prime}, k k^{\prime}}
$$

onto unpolarized gluons $(g)$, longitudinally polarized gluons $(\Delta g)$ and linearly polarized gluons $(\delta g)$. The tensor

$$
\tau^{j j^{\prime}, k k^{\prime}}=\frac{1}{2}\left(\delta^{j k} \delta^{j^{\prime} k^{\prime}}+\delta^{j k^{\prime}} \delta^{j^{\prime} k}-\delta^{j j^{\prime}} \delta^{k k^{\prime}}\right)
$$


satisfies $\tau^{j j^{\prime}, k k^{\prime}} \tau^{k k^{\prime}, l l^{\prime}}=\tau^{j j^{\prime}, l l^{\prime}}$ and is symmetric and traceless in each of the index pairs $\left(j j^{\prime}\right)$ and $\left(k k^{\prime}\right)$. Note that for gluons $\delta g$ denotes linear polarization, i.e. the interference between gluons whose helicities differ by two units in the scattering amplitude and its conjugate, while for quarks $\delta q$ symbolizes transverse polarization, where the interference is between quarks with a helicity difference of one unit. Since we limit ourselves to color-singlet distributions, a sum over the color indices of the quark fields in (2.2) and the gluon fields in (2.4) is implied. We do not write out the Wilson lines that make the operators gauge invariant.

The different spin projections lead to a large number of DPDs. For collinear colorsinglet distributions, several polarization combinations are zero due to time reversal and parity invariance. This concerns the DPDs with one longitudinally polarized and one unpolarized parton, as well as those with one longitudinally polarized parton and one transversely polarized (anti)quark or linearly polarized gluon. A decomposition of the nonzero distributions for two quarks in terms of real-valued scalar functions has already been given in [12]:

$$
\begin{aligned}
F_{q q}\left(x_{1}, x_{2}, \boldsymbol{y}\right) & =f_{q q}\left(x_{1}, x_{2}, y\right), \\
F_{\Delta q \Delta q}\left(x_{1}, x_{2}, \boldsymbol{y}\right) & =f_{\Delta q \Delta q}\left(x_{1}, x_{2}, y\right), \\
F_{q \delta q}^{j}\left(x_{1}, x_{2}, \boldsymbol{y}\right) & =\tilde{\boldsymbol{y}}^{j} M f_{q \delta q}\left(x_{1}, x_{2}, y\right), \\
F_{\delta q q}^{j}\left(x_{1}, x_{2}, \boldsymbol{y}\right) & =\tilde{\boldsymbol{y}}^{j} M f_{\delta q q}\left(x_{1}, x_{2}, y\right), \\
F_{\delta q \delta q}^{j j^{\prime}}\left(x_{1}, x_{2}, \boldsymbol{y}\right) & =\delta^{j j^{\prime}} f_{\delta q \delta q}\left(x_{1}, x_{2}, y\right)+2 \tau^{j j^{\prime}, \boldsymbol{y} \boldsymbol{y}} M^{2} f_{\delta q \delta q}^{t}\left(x_{1}, x_{2}, y\right),
\end{aligned}
$$

where $M$ is the proton mass, $\tilde{\boldsymbol{y}}^{j}=\epsilon^{j j^{\prime}} \boldsymbol{y}^{j^{\prime}}$ and $y=\sqrt{\boldsymbol{y}^{2}}$. We use a shorthand notation where vectors $\boldsymbol{y}$ or $\tilde{\boldsymbol{y}}$ appearing as an index of $\tau$ denote contraction, i.e. $\tau^{j j^{\prime}, \boldsymbol{y} \boldsymbol{y}}=\tau^{j j^{\prime}, k k^{\prime}} \boldsymbol{y}^{k} \boldsymbol{y}^{k^{\prime}}$ etc. Decompositions analogous to (2.7) hold for quark-antiquark distributions and for the distributions of two antiquarks.

Since quarks and gluons mix under evolution, we also need to consider DPDs involving gluons. We define

$$
\begin{aligned}
F_{q g}\left(x_{1}, x_{2}, \boldsymbol{y}\right) & =f_{q g}\left(x_{1}, x_{2}, y\right) \\
F_{\Delta q \Delta g}\left(x_{1}, x_{2}, \boldsymbol{y}\right)= & f_{\Delta q \Delta g}\left(x_{1}, x_{2}, y\right), \\
F_{q \delta g}^{j j^{\prime}}\left(x_{1}, x_{2}, \boldsymbol{y}\right)= & \tau^{j j^{\prime}, \boldsymbol{y} \boldsymbol{y}} M^{2} f_{q \delta g}\left(x_{1}, x_{2}, y\right) \\
F_{\delta q g}^{j}\left(x_{1}, x_{2}, \boldsymbol{y}\right)= & \tilde{\boldsymbol{y}}^{j} M f_{\delta q g}\left(x_{1}, x_{2}, y\right) \\
F_{\delta q \delta g}^{j, k k^{\prime}}\left(x_{1}, x_{2}, \boldsymbol{y}\right)= & -\tau^{\tilde{\boldsymbol{y}} j, k k^{\prime}} M f_{\delta q \delta g}\left(x_{1}, x_{2}, y\right) \\
& -\left(\tilde{\boldsymbol{y}}^{j} \tau^{k k^{\prime}, \boldsymbol{y} \boldsymbol{y}}+\boldsymbol{y}^{j} \tau^{k k^{\prime}, \boldsymbol{y} \tilde{\boldsymbol{y}}}\right) M^{3} f_{\delta q \delta g}^{t}\left(x_{1}, x_{2}, y\right)
\end{aligned}
$$

for quark-gluon distributions, with analogous expressions for gluon-quark distributions and distributions where the quark is replaced by an antiquark. For two-gluon distributions we write

$$
\begin{aligned}
F_{g g}\left(x_{1}, x_{2}, \boldsymbol{y}\right) & =f_{g g}\left(x_{1}, x_{2}, y\right), \\
F_{\Delta g \Delta g}\left(x_{1}, x_{2}, \boldsymbol{y}\right) & =f_{\Delta g \Delta g}\left(x_{1}, x_{2}, y\right), \\
F_{g \delta g}^{j j^{\prime}}\left(x_{1}, x_{2}, \boldsymbol{y}\right) & =\tau^{j j^{\prime}, \boldsymbol{y y}} M^{2} f_{g \delta g}\left(x_{1}, x_{2}, y\right),
\end{aligned}
$$




$$
\begin{aligned}
& F_{\delta g g}^{j j^{\prime}}\left(x_{1}, x_{2}, \boldsymbol{y}\right)=\tau^{j j^{\prime}, \boldsymbol{y y}} M^{2} f_{\delta g g}\left(x_{1}, x_{2}, y\right), \\
& F_{\delta g \delta g}^{j j^{\prime}, k k^{\prime}}\left(x_{1}, x_{2}, \boldsymbol{y}\right)=\frac{1}{2} \tau^{j j^{\prime}, k k^{\prime}} f_{\delta g \delta g}\left(x_{1}, x_{2}, y\right), \\
& +\left(\tau^{j j^{\prime}, \boldsymbol{y} \tilde{\boldsymbol{y}}} \tau^{k k^{\prime}, \boldsymbol{y} \tilde{\boldsymbol{y}}}-\tau^{j j^{\prime}, \boldsymbol{y} \boldsymbol{y}} \tau^{k k^{\prime}, \boldsymbol{y y}}\right) M^{4} f_{\delta g \delta g}^{t}\left(x_{1}, x_{2}, y\right) .
\end{aligned}
$$

We remark that, although linear gluon polarization is described by a tensor with two indices, the restriction that this tensor is symmetric and traceless gives rise to the same number of distributions as for transverse quark polarization, which is described by a vector. The prefactors in (2.8) and (2.9) have been chosen such that we will obtain a simple correspondence between quark and gluon distributions in the spin density matrices to be derived in the next section.

Note that DPDs involving gluons are not only relevant in the context of evolution but also enter directly in important double scattering processes such as the production of jets. Their properties are hence of considerable practical interest.

In complete analogy to the case of collinear single-parton distributions, the DPDs we have introduced can be interpreted as probability densities for finding two partons inside an unpolarized proton, with a relative transverse distance $\boldsymbol{y}$ and with longitudinal momentum fractions $x_{1}$ and $x_{2}$. This becomes evident from their appearance in the cross section formulae for double parton scattering [12]. It can also be seen from a representation in terms of parton creation and annihilation operators or from a representation in terms of the light-cone wave functions of the proton, which are straightforward extensions of the corresponding representations for single-parton distributions (given for instance in sections 3.4 and 3.11 of [19]).

As in the case of single-parton densities, this interpretation does however not strictly hold in QCD, because the distributions are defined with subtractions from the ultraviolet region of parton momenta. The subtraction terms can in principle invalidate the positivity of the distributions. Nevertheless, it is useful to explore the consequences of the probability interpretation as a guide for developing physically intuitive models of the distributions. This holds in particular if one works in leading order of $\alpha_{s}$, where the connection between parton distributions and physical cross sections (which must of course be positive semi-definite) is most direct.

\section{Two-parton spin density matrices}

The polarization state of two partons in an unpolarized proton is described by a spin density matrix that can be written in terms of the DPDs we introduced in the previous section. We start by trading the projection operators (2.3) and (2.5) for operators that project onto quarks or gluons of definite helicity. We can then easily write down the spin density matrix for two partons in the helicity basis.

The projection operators $\Gamma_{\lambda^{\prime} \lambda}$ for quarks, where $\lambda\left(\lambda^{\prime}\right)$ refers to the quark helicity in the amplitude (conjugate amplitude), are given by

$$
\Gamma_{++}=\frac{\gamma^{+}}{4}\left(1+\gamma_{5}\right)=\frac{\Gamma_{q}+\Gamma_{\Delta q}}{2}, \quad \Gamma_{+-}=\frac{i \sigma^{+1}}{4}\left(1-\gamma_{5}\right)=\frac{\Gamma_{\delta q}^{1}+i \Gamma_{\delta q}^{2}}{2}
$$




$$
\Gamma_{--}=\frac{\gamma^{+}}{4}\left(1-\gamma_{5}\right)=\frac{\Gamma_{q}-\Gamma_{\Delta q}}{2}, \quad \Gamma_{-+}=-\frac{i \sigma^{+1}}{4}\left(1+\gamma_{5}\right)=\frac{\Gamma_{\delta q}^{1}-i \Gamma_{\delta q}^{2}}{2} .
$$

Here we use the phase conventions for spin-half fields specified in [20]. The projection operators $\Pi_{\lambda^{\prime} \lambda}^{j j^{\prime}}$ for gluons, where $\lambda$ and $j\left(\lambda^{\prime}\right.$ and $\left.j^{\prime}\right)$ refer to the amplitude (conjugate amplitude), can be constructed from the polarization vectors

$$
\boldsymbol{\epsilon}_{+}=-\frac{1}{\sqrt{2}}(1, i), \quad \boldsymbol{\epsilon}_{-}=\frac{1}{\sqrt{2}}(1,-i)
$$

and read

$$
\begin{aligned}
& \Pi_{++}^{j j^{\prime}}=\left(\epsilon_{+}^{j}\right)^{*} \epsilon_{+}^{j^{\prime}}=\frac{1}{2}\left(\Pi_{g}^{j j^{\prime}}+\Pi_{\Delta g}^{j j^{\prime}}\right), \\
& \Pi_{--}^{j j^{\prime}}=\left(\epsilon_{-}^{j}\right)^{*} \epsilon_{-}^{j^{\prime}}=\frac{1}{2}\left(\Pi_{g}^{j j^{\prime}}-\Pi_{\Delta g}^{j j^{\prime}}\right), \\
& \Pi_{+-}^{j j^{\prime}}=\left(\epsilon_{-}^{j}\right)^{*} \epsilon_{+}^{j^{\prime}}=-\left[\Pi_{\delta g}^{11}\right]^{j j^{\prime}}-i\left[\Pi_{\delta g}^{12}\right]^{j j^{\prime}}, \\
& \Pi_{-+}^{j j^{\prime}}=\left(\epsilon_{+}^{j}\right)^{*} \epsilon_{-}^{j^{\prime}}=-\left[\Pi_{\delta g}^{11}\right]^{j j^{\prime}}+i\left[\Pi_{\delta g}^{12}\right]^{j j^{\prime}} .
\end{aligned}
$$

We can now organize the distributions in matrices where the columns (rows) correspond to helicity states,,,++-++--- of the two partons in the amplitude (conjugate amplitude). The spin density matrix for two quarks reads

$$
\rho=\frac{1}{4}\left(\begin{array}{cccc}
f_{q q}+f_{\Delta q \Delta q} & -i e^{i \varphi_{y}} y M f_{\delta q q} & -i e^{i \varphi_{y}} y M f_{q \delta q} & 2 e^{2 i \varphi_{y}} y^{2} M^{2} f_{\delta q \delta q}^{t} \\
i e^{-i \varphi_{y}} y M f_{\delta q q} & f_{q q}-f_{\Delta q \Delta q} & 2 f_{\delta q \delta q} & -i e^{i \varphi_{y}} y M f_{q \delta q} \\
i e^{-i \varphi_{y}} y M f_{q \delta q} & 2 f_{\delta q \delta q} & f_{q q}-f_{\Delta q \Delta q} & -i e^{i \varphi_{y}} y M f_{\delta q q} \\
2 e^{-2 i \varphi_{y}} y^{2} M^{2} f_{\delta q \delta q}^{t} & i e^{-i \varphi_{y}} y M f_{q \delta q} & i e^{-i \varphi_{y}} y M f_{\delta q q} & f_{q q}+f_{\Delta q \Delta q}
\end{array}\right)
$$

where the angle $\varphi_{y}$ describes the orientation of the vector $\boldsymbol{y}=y\left(\cos \varphi_{y}, \sin \varphi_{y}\right)$ in the transverse plane. With the caveat spelled out at the end of the previous section, the diagonal matrix elements can be interpreted as the probability densities for finding two partons in definite helicity states inside an unpolarized proton. Specifically, $f_{q q}+f_{\Delta q \Delta q}$ is the probability density for finding two quarks with positive helicities, which in an unpolarized proton is equal to the probability density for finding two quarks with negative helicities. The probability density for finding two quarks with opposite helicities is $f_{q q}-f_{\triangle q \Delta q}$. The off-diagonal elements of $\rho$ describe helicity interference, with $f_{\delta q \delta q}^{t}$ in the right upper corner corresponding for instance to the case where both quarks have negative helicity in the amplitude and positive helicity in the conjugate amplitude. This leads to a helicity difference between the amplitude and its conjugate, which is balanced by two units of orbital angular momentum indicated by an exponential $e^{2 i \varphi_{y}}$ and an associated factor $y^{2}$. By contrast, $f_{\delta q \delta q}$ describes the case when the helicity difference is +1 for one quark and -1 for the other, so that the overall helicity is balanced.

Turning now to gluons, we have a spin density matrix

$$
\frac{1}{4}\left(\begin{array}{cccc}
f_{q g}+f_{\Delta q \Delta g} & -i e^{i \varphi_{y}} y M f_{\delta q g} & -e^{2 i \varphi_{y}} y^{2} M^{2} f_{q \delta g} & -2 i e^{3 i \varphi_{y}} y^{3} M^{3} f_{\delta q \delta g}^{t} \\
i e^{-i \varphi_{y}} y M f_{\delta q g} & f_{q g}-f_{\Delta q \Delta g} & -2 i e^{i \varphi_{y}} y M f_{\delta q \delta g} & -e^{2 i \varphi_{y}} y^{2} M^{2} f_{q \delta g} \\
-e^{-2 i \varphi_{y}} y^{2} M^{2} f_{q \delta g} & 2 i e^{-i \varphi_{y}} y M f_{\delta q \delta g} & f_{q g}-f_{\Delta q \Delta g} & -i e^{i \varphi_{y}} y M f_{\delta q g} \\
2 i e^{-3 i \varphi_{y}} y^{3} M^{3} f_{\delta q \delta g}^{t} & -e^{-2 i \varphi_{y}} y^{2} M^{2} f_{q \delta g} & i e^{-i \varphi_{y}} y M f_{\delta q g} & f_{q g}+f_{\Delta q \Delta g}
\end{array}\right)
$$


for quark-gluon distributions and an analogous matrix for gluon-quark distributions. For two-gluon distributions we find

$$
\frac{1}{4}\left(\begin{array}{cccc}
f_{g g}+f_{\Delta g \Delta g} & -e^{2 i \varphi_{y}} y^{2} M^{2} f_{\delta g g} & -e^{2 i \varphi_{y}} y^{2} M^{2} f_{g \delta g} & -2 e^{4 i \varphi_{y}} y^{4} M^{4} f_{\delta g \delta g}^{t} \\
-e^{-2 i \varphi_{y}} y^{2} M^{2} f_{\delta g g} & f_{g g}-f_{\Delta g \Delta g} & 2 f_{\delta g \delta g} & -e^{2 i \varphi_{y}} y^{2} M^{2} f_{g \delta g} \\
-e^{-2 i \varphi_{y}} y^{2} M^{2} f_{g \delta g} & 2 f_{\delta g \delta g} & f_{g g}-f_{\Delta g \Delta g} & -e^{2 i \varphi_{y}} y^{2} M^{2} f_{\delta g g} \\
-2 e^{-4 i \varphi_{y}} y^{4} M^{4} f_{\delta g \delta g}^{t} & -e^{-2 i \varphi_{y}} y^{2} M^{2} f_{g \delta g} & -e^{-2 i \varphi_{y}} y^{2} M^{2} f_{\delta g g} & f_{g g}+f_{\Delta g \Delta g}
\end{array}\right) .
$$

The matrices for distributions where quarks are replaced by antiquarks are analogous to (3.4) and (3.5). We see that the parameterization of DPDs in the previous section gives simple expressions for the spin density matrices and similar structures for all types of partons.

The difference in spin between quarks and gluons causes the different dependence on the azimuthal angle $\varphi_{y}$ in (3.4), (3.5) and (3.6). A mismatch of $n$ units between the sum of parton helicities in the amplitude and its conjugate goes along with an exponential $e^{ \pm n i \varphi_{y}}$ and an associated factor $y^{n}$.

\section{Positivity bounds}

We now show how the probability interpretation of DPDs constrains the size of the polarized distributions. Since the probability density for finding two partons in a general polarization state is positive semi-definite, we have

$$
\sum_{\lambda_{1}^{\prime} \lambda_{2}^{\prime} \lambda_{1} \lambda_{2}} v_{\lambda_{1}^{\prime} \lambda_{2}^{\prime}}^{*} \rho_{\left(\lambda_{1}^{\prime} \lambda_{2}^{\prime}\right)\left(\lambda_{1} \lambda_{2}\right)} v_{\lambda_{1} \lambda_{2}} \geq 0
$$

with arbitrary complex coefficients $v_{\lambda_{1} \lambda_{2}}$ normalized as $\sum_{\lambda_{1} \lambda_{2}}\left|v_{\lambda_{1} \lambda_{2}}\right|^{2}=1$. The helicity matrices are therefore positive semi-definite. The same property has been derived for the spin density matrices associated with transverse-momentum dependent distributions [17] or generalized parton distributions [18].

To simplify the algebra, we first cast all helicity matrices into a common form that is independent of the angle $\varphi_{y}$. This is achieved by unitary transformations, multiplying by a matrix $U$ from the right and by $U^{\dagger}$ from the left. The transformation matrices for the parton combinations in (3.4) to (3.6) are

$$
\begin{aligned}
& U_{q q}=\operatorname{diag}\left(-e^{2 i \varphi_{y}},-i e^{i \varphi_{y}},-i e^{i \varphi_{y}}, 1\right), \\
& U_{q g}=\operatorname{diag}\left(i e^{3 i \varphi_{y}},-e^{2 i \varphi_{y}},-i e^{i \varphi_{y}}, 1\right), \\
& U_{g g}=\operatorname{diag}\left(e^{4 i \varphi_{y}},-e^{2 i \varphi_{y}},-e^{2 i \varphi_{y}}, 1\right) .
\end{aligned}
$$

After these transformations and their analog for gluon-quark distributions, the spin density matrices can be written as

$$
\rho=\frac{1}{4}\left(\begin{array}{cccc}
f_{a b}+f_{\Delta a \Delta b} & h_{\delta a b} & h_{a \delta b} & -2 h_{\delta a \delta b}^{t} \\
h_{\delta a b} & f_{a b}-f_{\Delta a \Delta b} & 2 h_{\delta a \delta b} & h_{a \delta b} \\
h_{a \delta b} & 2 h_{\delta a \delta b} & f_{a b}-f_{\Delta a \Delta b} & h_{\delta a b} \\
-2 h_{\delta a \delta b}^{t} & h_{a \delta b} & h_{\delta a b} & f_{a b}+f_{\Delta a \Delta b}
\end{array}\right)
$$


with the following identification of distributions for different parton combinations:

$$
\begin{aligned}
f_{a b} & =f_{q q}, f_{q g}, f_{g q}, f_{g g}, \\
f_{\Delta a \Delta b} & =f_{\Delta q \Delta q}, f_{\Delta q \Delta g}, f_{\Delta g \Delta q}, f_{\Delta g \Delta g}, \\
h_{\delta a b} & =y M f_{\delta q q}, y M f_{\delta q g}, y^{2} M^{2} f_{\delta g q}, y^{2} M^{2} f_{\delta g g}, \\
h_{a \delta b} & =y M f_{q \delta q}, y^{2} M^{2} f_{q \delta g}, y M f_{g \delta q}, y^{2} M^{2} f_{g \delta g}, \\
h_{\delta a \delta b} & =f_{\delta q \delta q}, y M f_{\delta q \delta g}, y M f_{\delta g \delta q}, f_{\delta g \delta g}, \\
h_{\delta a \delta b}^{t} & =y^{2} M^{2} f_{\delta q \delta q}^{t}, y^{3} M^{3} f_{\delta q \delta g}^{t}, y^{3} M^{3} f_{\delta g \delta q}^{t}, y^{4} M^{4} f_{\delta g \delta g}^{t} .
\end{aligned}
$$

Analogous expressions hold if quarks are replaced by antiquarks. Positivity ${ }^{1}$ of the diagonal elements of $\rho$ yields the trivial bounds

$$
f_{a b} \geq\left|f_{\Delta a \Delta b}\right|
$$

The principal minors of the two-dimensional sub-spaces must be positive semi-definite as well, which gives upper bounds on the distributions for one or two transversely or linearly polarized partons:

$$
\begin{aligned}
f_{a b}+f_{\Delta a \Delta b} & \geq 2\left|h_{\delta a \delta b}^{t}\right|, \\
f_{a b}-f_{\Delta a \Delta b} & \geq 2\left|h_{\delta a \delta b}\right|, \\
f_{a b}^{2} \geq\left(f_{a b}+f_{\Delta a \Delta b}\right)\left(f_{a b}-f_{\Delta a \Delta b}\right) & \geq h_{\delta a b}^{2}, \\
f_{a b}^{2} \geq\left(f_{a b}+f_{\Delta a \Delta b}\right)\left(f_{a b}-f_{\Delta a \Delta b}\right) & \geq h_{a \delta b}^{2} .
\end{aligned}
$$

The principal minors of dimension three, as well as $\operatorname{det}(\rho)$ provide further bounds, which are rather cumbersome and will not be given here. The strongest bounds can be obtained from the positivity of the eigenvalues of $\rho$, which is a sufficient and necessary condition for the positivity of $\rho$. Calculating the eigenvalues we obtain

$$
\begin{aligned}
& f_{a b}+h_{\delta a \delta b}-h_{\delta a \delta b}^{t} \pm \sqrt{\left(h_{\delta a b}+h_{a \delta b}\right)^{2}+\left(f_{\Delta a \Delta b}-h_{\delta a \delta b}-h_{\delta a \delta b}^{t}\right)^{2}} \geq 0 \\
& f_{a b}-h_{\delta a \delta b}+h_{\delta a \delta b}^{t} \pm \sqrt{\left(h_{\delta a b}-h_{a \delta b}\right)^{2}+\left(f_{\Delta a \Delta b}+h_{\delta a \delta b}+h_{\delta a \delta b}^{t}\right)^{2}} \geq 0 .
\end{aligned}
$$

These inequalities set upper limits on the size of spin correlations between two partons in an unpolarized proton. They can be used either to construct double parton distributions or to put limits on polarization effects in double hard scattering processes.

We note that positive semidefinite combinations of DPDs were discussed already in the pioneering studies $[9,10]$. Distributions that involve a helicity mismatch between the amplitude and its conjugate (see section 3) were however not considered in that work. The derivation in $[9,10]$ thus corresponds to our results (4.5) and (4.6) if all distributions multiplied with a power of $y$ in (4.4) are set to zero.

\footnotetext{
${ }^{1}$ For ease of language we use "positivity" in the sense of "positive semi-definite" here and in the following.
} 


\section{$5 \quad$ Stability under evolution}

The ultraviolet subtractions mentioned at the end of section 2 induce a scale dependence, which for collinear single-parton distributions is described by the DGLAP evolution equations. While the subtractions themselves may invalidate positivity of the distributions and thus their density interpretation, the evolution equations can be interpreted in a probabilistic manner provided that one takes the leading-order approximation of the evolution kernels $[23,24]$. Specifically, one finds that if parton distributions are positive semi-definite at a certain scale, this property is preserved by leading-order evolution to higher scales. This also holds for the Soffer inequality, which expresses positivity in the sector of transverse quark polarization $[25,26]$. For evolution at next-to-leading order in $\alpha_{s}$ the situation is less clear-cut and a discussion of positivity depends in particular on the scheme in which the distributions are defined. We refer to [27] and [28, 29] for a discussion of the situation for longitudinal and transverse parton polarization, respectively.

Returning to double parton distributions, we now show that the bounds derived in the previous section are stable under leading-order evolution to higher scales. The strategy for the derivation is as follows: we first introduce linear combinations of double parton distributions whose positivity is necessary and sufficient for the positivity of the spin density matrices and then show that these linear combinations remain positive semi-definite under evolution. The positivity of the spin density matrices then guarantees the stability of the positivity bounds.

\subsection{Evolution of double parton distributions}

To begin with, let us specify the evolution of collinear DPDs in the color-singlet sector. We use the homogeneous evolution equations in the transverse position representation, see e.g. equation (5.93) in [12]. These equations apply at nonzero $\boldsymbol{y}$ if $F_{a_{1} a_{2}}\left(x_{1}, x_{2}, \boldsymbol{y}\right)$ is defined via (2.1) with the operators $\mathcal{O}_{a_{1}}\left(y, z_{1}\right)$ and $\mathcal{O}_{a_{2}}\left(0, z_{2}\right)$ renormalized by standard $\overline{\mathrm{MS}}$ subtraction. The inhomogeneous term for the splitting of one parton into two that has been previously considered in the literature [30-34] does not appear in this case. As discussed in [11, 12], a consistent formulation of factorization for double parton scattering does not yet exist, so that it remains unclear how DPDs should best be defined (and how they evolve). For simplicity we will limit our present investigation to the homogeneous evolution equations.

It is useful for our purpose to take different renormalization scales $\mu_{1}$ and $\mu_{2}$ for the two partons, corresponding to separate ultraviolet renormalization of $\mathcal{O}_{a_{1}}$ and $\mathcal{O}_{a_{2}}$ in (2.1). The evolution equation for the unpolarized double quark distributions in the first scale then reads

$$
\frac{\partial f_{q q}\left(x_{1}, x_{2}, y ; \mu_{1}, \mu_{2}\right)}{\partial \tau_{1}}=P_{q q} \otimes_{1} f_{q q}+P_{q g} \otimes_{1} f_{g q}
$$

where

$$
P_{a b}(.) \otimes_{1} f_{b c}\left(., x_{2}, y ; \mu_{1}, \mu_{2}\right)=\int_{x_{1}}^{1-x_{2}} \frac{d u_{1}}{u_{1}} P_{a b}\left(\frac{x_{1}}{u_{1}}\right) f_{b c}\left(u_{1}, x_{2}, y ; \mu_{1}, \mu_{2}\right)
$$

is a convolution in the first argument of the DPDs with the leading-order splitting functions $P_{a b}$ known from DGLAP evolution of single-parton distributions. We note that the leading- 
order splitting functions are the same for quarks and antiquarks, i.e. one has $P_{q q}=P_{\bar{q} \bar{q}}$, $P_{q g}=P_{\bar{q} g}, P_{g q}=P_{g \bar{q}}$ and analogous relations for polarized partons. In appendix A we give the explicit evolution equations for all polarized DPDs and list the associated splitting functions.

The evolution variable in (5.1) is taken as

$$
\tau_{1}=\int^{\mu_{1}^{2}} \frac{d \mu^{2}}{\mu^{2}} \frac{\alpha_{s}(\mu)}{2 \pi},
$$

where the lower limit of integration is irrelevant in the derivative $\partial f / \partial \tau_{1}$. The use of $\tau_{1}$ is just a matter of convenience as it removes the running coupling from the leading-order splitting functions.

The analog of (5.1) for the scale associated with the second parton is

$$
\frac{\partial f_{q q}\left(x_{1}, x_{2}, y ; \mu_{1}, \mu_{2}\right)}{\partial \tau_{2}}=P_{q q} \otimes_{2} f_{q q}+P_{q g} \otimes_{2} f_{q g} .
$$

The evolution equation for equal scales, i.e. for $f_{q q}\left(x_{1}, x_{2}, y ; \mu, \mu\right)$, is readily obtained by adding the right-hand sides of (5.1) and (5.4). We will show that positivity is preserved for separate evolution in $\mu_{1}$. The same then obviously holds for evolution in $\mu_{2}$ and hence for the evolution in a single common scale $\mu_{1}=\mu_{2}$.

\subsection{Linear combinations of DPDs}

A key ingredient in our argument is to form suitable linear combinations of double parton distributions, which we now introduce. Positivity of the spin density matrix $\rho$ means that $v^{\dagger} \rho v \geq 0$ for any complex vector $v$, as we spelled out in (4.1). Parameterizing the vector as

$$
v^{T}=\left(a_{1}+i b_{1}, a_{2}+i b_{2}, a_{3}+i b_{3}, a_{4}+i b_{4}\right)
$$

with real numbers $a_{i}, b_{i}$ and performing the multiplication with the matrix in (4.3) gives

$$
Q_{a b}^{+}=c_{1} f_{a b}+c_{2} h_{a \delta b}+c_{3} f_{\Delta a \Delta b}+c_{4} h_{\delta a b}+c_{5} h_{\delta a \delta b}+c_{6} h_{\delta a \delta b}^{t} \geq 0,
$$

where $Q_{a b}^{+}=4 v^{\dagger} \rho v$ and the coefficients $c_{i}$ are given by

$$
\begin{array}{ll}
c_{1}=a_{1}^{2}+b_{1}^{2}+a_{2}^{2}+b_{2}^{2}+a_{3}^{2}+b_{3}^{2}+a_{4}^{2}+b_{4}^{2}, & c_{2}=2\left(a_{1} a_{3}+b_{1} b_{3}+a_{2} a_{4}+b_{2} b_{4}\right), \\
c_{3}=a_{1}^{2}+b_{1}^{2}-a_{2}^{2}-b_{2}^{2}-a_{3}^{2}-b_{3}^{2}+a_{4}^{2}+b_{4}^{2}, & c_{4}=2\left(a_{1} a_{2}+b_{1} b_{2}+a_{3} a_{4}+b_{3} b_{4}\right), \\
c_{5}=4\left(a_{2} a_{3}+b_{2} b_{3}\right), & c_{6}=-4\left(a_{1} a_{4}+b_{1} b_{4}\right) .
\end{array}
$$

We will prove the stability of the positivity bounds by showing that for arbitrary values of $a_{i}$ and $b_{i}$ the inequality (5.6) is stable under evolution to higher scales. It will be convenient to consider further linear combinations of double parton distributions. Changing signs of the parameters $a_{1} \rightarrow-a_{1}, b_{1} \rightarrow-b_{1}, a_{3} \rightarrow-a_{3}, b_{3} \rightarrow-b_{3}$ we get

$$
Q_{a b}^{-}=c_{1} f_{a b}+c_{2} h_{a \delta b}+c_{3} f_{\Delta a \Delta b}-c_{4} h_{\delta a b}-c_{5} h_{\delta a \delta b}-c_{6} h_{\delta a \delta b}^{t} \geq 0 .
$$


Adding (5.6) and (5.8) gives the simpler inequality

$$
B_{a b}^{+}=c_{1} f_{a b}+c_{2} h_{a \delta b}+c_{3} f_{\Delta a \Delta b} \geq 0,
$$

and interchanging indices $(1 \leftrightarrow 2$ and $3 \leftrightarrow 4)$ in the elements of $v$ gives

$$
B_{a b}^{-}=c_{1} f_{a b}+c_{2} h_{a \delta b}-c_{3} f_{\triangle a \Delta b} \geq 0 .
$$

If (5.6) holds at a given scale for arbitrary values of $a_{i}$ and $b_{i}$, then (5.8) to (5.10) hold at that scale as well.

We will see that the evolution equations in the scale $\mu_{1}$ can be formulated in terms of $Q_{a b}^{+}, Q_{a b}^{-}$and $B_{a b}^{-}$alone. ${ }^{2}$ This becomes plausible if we note that these three functions are linear combinations of $\left(c_{1} f_{a b}+c_{2} h_{a \delta b}\right), f_{\Delta a \Delta b}$ and $\left(c_{4} h_{\delta a b}+c_{5} h_{\delta a \delta b}+c_{6} h_{\delta a \delta b}^{t}\right)$ and that for evolution in $\mu_{1}$ only the polarization of the first parton is relevant but not the polarization of the second parton. The linear combinations $Q_{a b}^{ \pm}$may be regarded as generalizations of the distributions $Q_{ \pm}=\frac{1}{2}(q+\bar{q}) \pm \delta q$ introduced in [26], where it was shown that the Soffer bound for the quark transversity distribution $\delta q$ is stable under leading-order evolution to higher scales.

\subsection{Evolution of the linear combinations}

We now show that the distributions $Q_{a b}^{ \pm}$and $B_{a b}^{ \pm}$remain positive semi-definite under leading-order evolution to higher scales. This implies the positivity of the spin density matrices and thereby the validity of the bounds derived in section 4.

The evolution equations for the distributions $Q_{a b}^{ \pm}$are

$$
\begin{aligned}
\frac{\partial}{\partial \tau_{1}}\left(\begin{array}{c}
Q_{q b}^{+} \\
Q_{q b}^{-}
\end{array}\right)= & \left(\begin{array}{cc}
\delta P_{q q}^{+} & \delta P_{q q}^{-} \\
\delta P_{q q}^{-} & \delta P_{q q}^{+}
\end{array}\right) \otimes_{1}\left(\begin{array}{c}
Q_{q b}^{+} \\
Q_{q b}^{-}
\end{array}\right)+\left(\begin{array}{cc}
P_{q g}^{+} & P_{q g}^{-} \\
P_{q g}^{+} & P_{q g}^{-}
\end{array}\right) \otimes_{1}\left(\begin{array}{c}
B_{g b}^{+} \\
B_{g b}^{-}
\end{array}\right) \\
& +\left(\begin{array}{cc}
P_{q q}^{-} & P_{q q}^{-} \\
P_{q q}^{-} & P_{q q}^{-}
\end{array}\right) \otimes_{1}\left(\begin{array}{c}
B_{q b}^{+} \\
B_{q b}^{-}
\end{array}\right)
\end{aligned}
$$

for a quark as first parton and

$$
\begin{aligned}
\frac{\partial}{\partial \tau_{1}}\left(\begin{array}{c}
Q_{g b}^{+} \\
Q_{g b}^{-}
\end{array}\right)= & \left(\begin{array}{cc}
\delta P_{g g}^{+} & \delta P_{g g}^{-} \\
\delta P_{g g}^{-} & \delta P_{g g}^{+}
\end{array}\right) \otimes_{1}\left(\begin{array}{c}
Q_{g b}^{+} \\
Q_{g b}^{-}
\end{array}\right)+\sum_{a=q, \bar{q}}\left(\begin{array}{cc}
P_{g a}^{+} & P_{g a}^{-} \\
P_{g a}^{+} & P_{g a}^{-}
\end{array}\right) \otimes_{1}\left(\begin{array}{c}
B_{a b}^{+} \\
B_{a b}^{-}
\end{array}\right) \\
& +\left(\begin{array}{cc}
P_{g g}^{-} & P_{g g}^{-} \\
P_{g g}^{-} & P_{g g}^{-}
\end{array}\right) \otimes_{1}\left(\begin{array}{c}
B_{g b}^{+} \\
B_{g b}^{-}
\end{array}\right)
\end{aligned}
$$

when the first parton is a gluon. The evolution equations for $B_{a b}^{ \pm}$read

$$
\frac{\partial}{\partial \tau_{1}}\left(\begin{array}{c}
B_{q b}^{+} \\
B_{q b}^{-}
\end{array}\right)=\left(\begin{array}{cc}
P_{q q}^{+} & P_{q q}^{-} \\
P_{q q}^{-} & P_{q q}^{+}
\end{array}\right) \otimes_{1}\left(\begin{array}{c}
B_{q b}^{+} \\
B_{q b}^{-}
\end{array}\right)+\left(\begin{array}{cc}
P_{q g}^{+} & P_{q g}^{-} \\
P_{q g}^{-} & P_{q g}^{+}
\end{array}\right) \otimes_{1}\left(\begin{array}{c}
B_{g b}^{+} \\
B_{g b}^{-}
\end{array}\right)
$$

\footnotetext{
${ }^{2}$ The combination $B_{a b}^{+}=\left(Q_{a b}^{+}+Q_{a b}^{-}\right) / 2$ is not independent and just used as an abbreviation.
} 
for a quark and

$$
\frac{\partial}{\partial \tau_{1}}\left(\begin{array}{c}
B_{g b}^{+} \\
B_{g b}^{-}
\end{array}\right)=\left(\begin{array}{cc}
P_{g g}^{+} & P_{g g}^{-} \\
P_{g g}^{-} & P_{g g}^{+}
\end{array}\right) \otimes_{1}\left(\begin{array}{c}
B_{g b}^{+} \\
B_{g b}^{-}
\end{array}\right)+\sum_{a=q, \bar{q}}\left(\begin{array}{cc}
P_{g a}^{+} & P_{g a}^{-} \\
P_{g a}^{-} & P_{g a}^{+}
\end{array}\right) \otimes_{1}\left(\begin{array}{c}
B_{a b}^{+} \\
B_{a b}^{-}
\end{array}\right)
$$

for a gluon. The evolution equations have the same form for antiquarks, i.e. (5.11) and (5.13) remain valid if we replace $q \rightarrow \bar{q}$ everywhere (except in the label $b$ for the second parton, which always remains fixed when we consider evolution in $\mu_{1}$ ).

The splitting functions appearing in the above equations are defined as

$$
P_{a b}^{ \pm}=\frac{1}{2}\left(P_{a b} \pm P_{\Delta a \Delta b}\right), \quad \delta P_{a b}^{ \pm}=\frac{1}{2}\left(P_{\Delta a \Delta b} \pm P_{\delta a \delta b}\right)
$$

for all parton indices $a$ and $b$. We remark that the kernels $P_{a b}^{+}\left(P_{a b}^{-}\right)$correspond to the case where the parton helicity is conserved (flipped). The only splitting functions that receive contributions from virtual graphs and hence contain a plus-prescription or an explicit $\delta$ function are

$$
\begin{aligned}
P_{q q}^{+} & =\frac{C_{F}}{2}\left[\frac{2\left(1+z^{2}\right)}{(1-z)_{+}}+3 \delta(1-z)\right], \\
\delta P_{q q}^{+} & =\frac{C_{F}}{2}\left[\frac{(1+z)^{2}}{(1-z)_{+}}+3 \delta(1-z)\right], \\
P_{g g}^{+} & =2 N_{c}\left[\frac{z}{(1-z)_{+}}+\frac{(1-z)(1+z)^{2}}{2 z}\right]+\frac{\beta_{0}}{2} \delta(1-z), \\
\delta P_{g g}^{+} & =2 N_{c}\left[\frac{z}{(1-z)_{+}}+1-z\right]+\frac{\beta_{0}}{2} \delta(1-z)
\end{aligned}
$$

with $N_{c}=3, C_{F}=4 / 3$ and

$$
\beta_{0}=\frac{11}{3} N_{c}-\frac{2}{3} n_{f},
$$

where $n_{f}$ is the number of active quark flavors. They are all positive for $0<z<1$ but have negative contributions at $z=1$ that arise from the plus-prescription, whose form is recalled in (B.3). In appendix B we show explicitly that the virtual contribution to evolution cannot change the sign of the distributions, which has previously been argued to be the case based on the probabilistic interpretation of leading-order evolution and its relation to the Boltzmann equation $[23,24,26]$. The reason for this property is that the virtual contribution to the evolution of a function is proportional to the function itself. We can then conclude that the diagonal terms in the evolution equations (5.11) to (5.14) preserve positivity. The off-diagonal kernels

$$
\begin{aligned}
P_{q q}^{-} & =0, & P_{g g}^{-} & =N_{c}(1-z)^{3} / z, \\
\delta P_{q q}^{-} & =C_{F}(1-z) / 2, & \delta P_{g g}^{-} & =2 N_{c}(1-z)
\end{aligned}
$$

and

$$
\begin{aligned}
& P_{q g}^{+}=z^{2} / 2, \\
& P_{g q}^{+}=C_{F} / z, \\
& P_{q g}^{-}=(1-z)^{2} / 2, \\
& P_{g q}^{-}=C_{F}(1-z)^{2} / z \text {. }
\end{aligned}
$$


are all positive or zero for $0<z<1$ and regular at $z=1$. Therefore they only reinforce positivity. In summary, if we have positive semi-definite initial conditions for all functions $Q_{a b}^{ \pm}$and $B_{a b}^{ \pm}$at some scale, then evolution to higher scales preserves this property. A more explicit derivation is given in appendix B.

\section{Conclusions}

We have derived spin density matrices for double parton distributions of quarks, anti-quarks and gluons. These matrices reveal the full polarization structure of two partons in an unpolarized proton and show the correspondence between the different polarized double parton distributions and parton helicities. The probabilistic interpretation of the double parton distribution for an arbitrary polarization state of the two partons gives upper limits on the size of spin correlations. These positivity bounds can be useful for modeling the otherwise poorly constrained double parton distributions and for deriving upper limits on spin effects in double hard scattering processes. We have shown that the homogeneous leading-order evolution equations preserve the bounds when going from lower to higher scales.

\section{A Evolution equations and splitting functions}

For completeness we give here the leading-order evolution equations for the first parton in the double parton distributions. When the first parton is a quark, we have

$$
\begin{aligned}
\frac{\partial f_{q b}}{\partial \tau_{1}} & =P_{q q} \otimes_{1} f_{q b}+P_{q g} \otimes_{1} f_{g b}, \\
\frac{\partial f_{q \delta b}}{\partial \tau_{1}} & =P_{q q} \otimes_{1} f_{q \delta b}+P_{q g} \otimes_{1} f_{g \delta b}, \\
\frac{\partial f_{\Delta q \Delta b}}{\partial \tau_{1}} & =P_{\Delta q \Delta q} \otimes_{1} f_{\Delta q \Delta b}+P_{\Delta q \Delta g} \otimes_{1} f_{\Delta g \Delta b}, \\
\frac{\partial f_{\delta q b}}{\partial \tau_{1}} & =P_{\delta q \delta q} \otimes_{1} f_{\delta q b}, \quad \frac{\partial f_{\delta q \delta b}}{\partial \tau_{1}}=P_{\delta q \delta q} \otimes_{1} f_{\delta q \delta b}, \quad \frac{\partial f_{\delta q \delta b}^{t}}{\partial \tau_{1}}=P_{\delta q \delta q} \otimes_{1} f_{\delta q \delta b}^{t}
\end{aligned}
$$

for $b=q, \bar{q}, g$. The arguments of the distributions are as in (5.1) and (5.2). Analogous equations hold if the first parton is an antiquark. For gluons we have

$$
\begin{aligned}
\frac{\partial f_{g b}}{\partial \tau_{1}} & =P_{g g} \otimes_{1} f_{g b}+\sum_{a=q, \bar{q}} P_{g a} \otimes_{1} f_{a b}, \\
\frac{\partial f_{g \delta b}}{\partial \tau_{1}} & =P_{g g} \otimes_{1} f_{g \delta b}+\sum_{a=q, \bar{q}} P_{g a} \otimes_{1} f_{a \delta b}, \\
\frac{\partial f_{\Delta g \Delta b}}{\partial \tau_{1}} & =P_{\Delta g \Delta g} \otimes_{1} f_{\Delta g \Delta b}+\sum_{a=q, \bar{q}} P_{\Delta g \Delta a} \otimes_{1} f_{\Delta a \Delta b}, \\
\frac{\partial f_{\delta g b}}{\partial \tau_{1}} & =P_{\delta g \delta g} \otimes_{1} f_{\delta g b}, \quad \frac{\partial f_{\delta g \delta b}}{\partial \tau_{1}}=P_{\delta g \delta g} \otimes_{1} f_{\delta g \delta b}, \quad \frac{\partial f_{\delta g \delta b}^{t}}{\partial \tau_{1}}=P_{\delta g \delta g} \otimes_{1} f_{\delta g \delta b}^{t} .
\end{aligned}
$$

The leading-order splitting functions have been derived in [21, 22]. They are given by

$$
P_{q q}(z)=C_{F}\left[\frac{1+z^{2}}{(1-z)_{+}}+\frac{3}{2} \delta(1-z)\right],
$$




$$
\begin{aligned}
P_{\Delta q \Delta q}(z) & =P_{q q}(z) \\
P_{\delta q \delta q}(z) & =P_{q q}(z)-C_{F}(1-z)
\end{aligned}
$$

for quark-quark transitions and by

$$
\begin{aligned}
P_{g g}(z) & =2 N_{c}\left[\frac{z}{(1-z)_{+}}+\frac{(1-z)\left(1+z^{2}\right)}{z}\right]+\frac{\beta_{0}}{2} \delta(1-z), \\
P_{\Delta g \Delta g}(z) & =P_{g g}(z)-2 N_{c} \frac{(1-z)^{3}}{z}, \\
P_{\delta g \delta g}(z) & =P_{g g}(z)-2 N_{c} \frac{(1-z)\left(1+z^{2}\right)}{z}
\end{aligned}
$$

for gluons. The splitting functions that mix quarks and gluons read

$$
\begin{aligned}
P_{q g} & =\frac{z^{2}+(1-z)^{2}}{2}, & P_{g q} & =C_{F} \frac{1+(1-z)^{2}}{z}, \\
P_{\Delta q \Delta g} & =\frac{z^{2}-(1-z)^{2}}{2}, & P_{\Delta g \Delta q} & =C_{F} \frac{1-(1-z)^{2}}{z} .
\end{aligned}
$$

As already mentioned below (5.2), the splitting functions are identical for quarks and antiquarks, i.e. (A.3) and (A.5) remain valid if we replace $q \rightarrow \bar{q}$. At leading order in $\alpha_{s}$ there are no direct transitions between quarks and antiquarks.

\section{B Elements of a stability proof}

In this appendix we show in more detail that the evolution equations in section 5.3 preserve positivity, taking particular care of the negative terms in the splitting functions that arise from virtual graphs and are implicit in the plus-prescription. We first consider the evolution of a single distribution and then extend the argument to the full coupled system of evolution equations.

We examine a function evolving as

$$
\frac{\partial}{\partial \tau} f(x, \tau)=\int_{x}^{v} \frac{d u}{u} P\left(\frac{x}{u}\right) f(u, \tau)
$$

with $0<x<v \leq 1$ and separate the splitting function as

$$
P(z)=\frac{P_{s}(z)}{(1-z)_{+}}+P_{r}(z)+P_{\delta} \delta(1-z)
$$

where $P_{s}(z)$ and $P_{r}(z)$ are positive semi-definite for $0<z<1$ and regular at $z=1$. The constant $P_{\delta}$ may be positive, negative or zero. The plus-prescription is defined as usual by

$$
[s(z)]_{+}=s(z)-\delta(1-z) \int_{0}^{1} d z^{\prime} s\left(z^{\prime}\right),
$$

where it is understood that the non-integrable singularity in the last term cancels when (B.3) is integrated over with a smooth test function. The plus-prescription part 
of the convolution in (B.1) can be written as

$$
\begin{aligned}
& \int_{x}^{v} \frac{d u}{u} \frac{P_{s}(x / u)}{(1-x / u)_{+}} f(u, \tau) \\
& \quad=\int_{x+\epsilon}^{v} d u \frac{P_{s}(x / u)}{u-x} f(u, \tau)+\int_{0}^{x-\epsilon} d u \frac{P_{s}(1)}{u-x} f(x, \tau)+\mathcal{O}(\epsilon),
\end{aligned}
$$

where for the error estimate we have assumed that $f(u, \tau)$ is differentiable at $u=x$. Defining

$$
\begin{aligned}
g_{\epsilon}(x, \tau ; f) & =\int_{x+\epsilon}^{v} d u\left[\frac{P_{s}(x / u)}{u-x}+\frac{P_{r}(x / u)}{u}\right] f(u, \tau), \\
h_{\epsilon}(x) & =-P_{\delta}+P_{s}(1) \int_{0}^{x-\epsilon} \frac{d u}{x-u}
\end{aligned}
$$

we can approximate the evolution of $f$ by

$$
\frac{\partial}{\partial \tau} f(x, \tau)=g_{\epsilon}(x, \tau ; f)-h_{\epsilon}(x) f(x, \tau)
$$

with an error that becomes arbitrarily small for $\epsilon \rightarrow 0$. In a more formal proof, one would replace $f$ with $f_{\epsilon}$ in (B.6) and show that $\lim _{\epsilon \rightarrow 0} f_{\epsilon}$ is a solution of (B.1). We now rewrite (B.6) as

$$
\frac{\partial}{\partial \tau}\left[e^{\tau h_{\epsilon}(x)} f(x, \tau)\right]=e^{\tau h_{\epsilon}(x)} g_{\epsilon}(x, \tau ; f) .
$$

Since $g_{\epsilon}$ is the convolution of $f(x, \tau)$ with a positive semi-definite integral kernel, the r.h.s. of this equation is positive semi-definite as long as $f(x, \tau)$ is. With initial conditions $f\left(x, \tau_{0}\right) \geq 0$ for all $x$ at a starting scale $\tau_{0}$, the function $e^{\tau h_{\epsilon}(x)} f(x, \tau)$ can therefore not decrease as $\tau$ increases, so that $f(x, \tau)$ stays positive semi-definite for all $\tau>\tau_{0}$. We note that the sign of $h_{\epsilon}(x)$ and thus of the constant $P_{\delta}$ is irrelevant for this argument.

We now consider the coupled system of evolution equations given by (5.11) to (5.14). Using a vector notation $f^{i}(x, \tau)$ for the $8 n_{f}+4$ functions $Q_{a b}^{+}, Q_{a b}^{-}, B_{a b}^{+}, B_{a b}^{-}$with $a=q, \bar{q}, g$ (and $b$ fixed), we can cast their evolution into the form

$$
\frac{\partial}{\partial \tau} f^{i}(x, \tau)=g_{\epsilon}^{i}\left(x, \tau ; f^{i}\right)-h_{\epsilon}^{i}(x) f^{i}(x, \tau)+\sum_{i \neq j} \int_{x}^{v} \frac{d u}{u} P^{i j}\left(\frac{x}{u}\right) f^{j}(u, \tau)
$$

with $i=1, \ldots, 8 n_{f}+4$. Here $g_{\epsilon}^{i}$ and $h_{\epsilon}^{i}$ are defined as in (B.5) with regular and positive semi-definite functions $P_{s}^{i}(z)$ and $P_{r}^{i}(z)$. The mixing kernels $P^{i j}(z)$ in (B.8) are regular and positive semi-definite as well. Rewriting the evolution as

$$
\frac{\partial}{\partial \tau}\left[e^{\tau h_{\epsilon}(x)} f^{i}(x, \tau)\right]=e^{\tau h_{\epsilon}(x)}\left[g_{\epsilon}^{i}\left(x, \tau ; f^{i}\right)+\sum_{i \neq j} \int_{x}^{v} \frac{d u}{u} P^{i j}\left(\frac{x}{u}\right) f^{j}(u, \tau)\right]
$$

we see that if one has initial conditions $f^{j}\left(x, \tau_{0}\right) \geq 0$ for all $j$ then all functions $f^{j}(x, \tau)$ remain positive semi-definite for $\tau>\tau_{0}$. 
Open Access. This article is distributed under the terms of the Creative Commons Attribution License which permits any use, distribution and reproduction in any medium, provided the original author(s) and source are credited.

\section{References}

[1] G. Calucci and D. Treleani, Mini-jets and the two-body parton correlation, Phys. Rev. D 57 (1998) 503 [hep-ph/9707389] [INSPIRE].

[2] G. Calucci and D. Treleani, Proton structure in transverse space and the effective cross-section, Phys. Rev. D 60 (1999) 054023 [hep-ph/9902479] [INSPIRE].

[3] A. Del Fabbro and D. Treleani, Scale factor in double parton collisions and parton densities in transverse space, Phys. Rev. D 63 (2001) 057901 [hep-ph/0005273] [INSPIRE].

[4] C. Flensburg, G. Gustafson, L. Lönnblad and A. Ster, Correlations in double parton distributions at small $x$, JHEP 06 (2011) 066 [arXiv:1103.4320] [INSPIRE].

[5] T. Rogers and M. Strikman, Multiple hard partonic collisions with correlations in proton-proton scattering, Phys. Rev. D 81 (2010) 016013 [arXiv:0908.0251] [INSPIRE].

[6] R. Corke and T. Sjöstrand, Multiparton interactions with an x-dependent proton size, JHEP 05 (2011) 009 [arXiv: 1101.5953] [INSPIRE].

[7] S. Domdey, H.-J. Pirner and U.A. Wiedemann, Testing the scale dependence of the scale factor $\sigma_{\mathrm{eff}}$ in double dijet production at the LHC, Eur. Phys. J. C 65 (2010) 153 [arXiv:0906.4335] [INSPIRE].

[8] M. Rinaldi, S. Scopetta and V. Vento, Double parton correlations in constituent quark models, arXiv: 1302.6462 [INSPIRE].

[9] M. Mekhfi, Correlations in color and spin in multiparton processes, Phys. Rev. D 32 (1985) 2380 [InSPIRE].

[10] M. Mekhfi, Multiparton processes: an application to double Drell-Yan, Phys. Rev. D 32 (1985) 2371 [INSPIRE].

[11] M. Diehl and A. Schäfer, Theoretical considerations on multiparton interactions in $Q C D$, Phys. Lett. B 698 (2011) 389 [arXiv:1102.3081] [INSPIRE].

[12] M. Diehl, D. Ostermeier and A. Schäfer, Elements of a theory for multiparton interactions in QCD, JHEP 03 (2012) 089 [arXiv:1111.0910] [INSPIRE].

[13] A.V. Manohar and W.J. Waalewijn, A QCD analysis of double parton scattering: color correlations, interference effects and evolution, Phys. Rev. D 85 (2012) 114009 [arXiv: 1202.3794] [INSPIRE].

[14] T. Kasemets and M. Diehl, Angular correlations in the double Drell-Yan process, JHEP 01 (2013) 121 [arXiv:1210.5434] [INSPIRE].

[15] H.-M. Chang, A.V. Manohar and W.J. Waalewijn, Double parton correlations in the bag model, Phys. Rev. D 87 (2013) 034009 [arXiv:1211.3132] [INSPIRE].

[16] J. Soffer, Positivity constraints for spin dependent parton distributions, Phys. Rev. Lett. 74 (1995) 1292 [hep-ph/9409254] [INSPIRE]. 
[17] A. Bacchetta, M. Boglione, A. Henneman and P. Mulders, Bounds on transverse momentum dependent distribution and fragmentation functions, Phys. Rev. Lett. 85 (2000) 712 [hep-ph/9912490] [INSPIRE].

[18] M. Diehl and P. Hägler, Spin densities in the transverse plane and generalized transversity distributions, Eur. Phys. J. C 44 (2005) 87 [hep-ph/0504175] [INSPIRE].

[19] M. Diehl, Generalized parton distributions, Phys. Rept. 388 (2003) 41 [hep-ph/0307382] [INSPIRE].

[20] M. Diehl, Generalized parton distributions with helicity flip, Eur. Phys. J. C 19 (2001) 485 [hep-ph/0101335] [INSPIRE].

[21] G. Altarelli and G. Parisi, Asymptotic freedom in parton language, Nucl. Phys. B 126 (1977) 298 [InSPIRE].

[22] X. Artru and M. Mekhfi, Transversely polarized parton densities, their evolution and their measurement, Z. Phys. C 45 (1990) 669 [INSPIRE].

[23] L. Durand and W. Putikka, Probabilistic derivation of parton splitting functions, Phys. Rev. D 36 (1987) 2840 [INSPIRE].

[24] J.C. Collins and J.-W. Qiu, A new derivation of the Altarelli-Parisi equations, Phys. Rev. D 39 (1989) 1398 [INSPIRE].

[25] V. Barone, On the QCD evolution of the transversity distribution, Phys. Lett. B 409 (1997) 499 [hep-ph/9703343] [INSPIRE].

[26] C. Bourrely, J. Soffer and O. Teryaev, The $Q^{2}$ evolution of Soffer inequality, Phys. Lett. B 420 (1998) 375 [hep-ph/9710224] [INSPIRE].

[27] G. Altarelli, S. Forte and G. Ridolfi, On positivity of parton distributions, Nucl. Phys. B 534 (1998) 277 [hep-ph/9806345] [INSPIRE].

[28] W. Vogelsang, Next-to-leading order evolution of transversity distributions and Soffer's inequality, Phys. Rev. D 57 (1998) 1886 [hep-ph/9706511] [INSPIRE].

[29] O. Martin, A. Schäfer, M. Stratmann and W. Vogelsang, Soffer's inequality and the transversely polarized Drell-Yan process at next-to-leading order, Phys. Rev. D 57 (1998) 3084 [hep-ph/9710300] [INSPIRE].

[30] R. Kirschner, Generalized Lipatov-Altarelli-Parisi equations and jet calculus rules, Phys. Lett. B 84 (1979) 266 [InSPIRE].

[31] V. Shelest, A. Snigirev and G. Zinovev, The multiparton distribution equations in QCD, Phys. Lett. B 113 (1982) 325 [INSPIRE].

[32] A. Snigirev, Double parton distributions in the leading logarithm approximation of perturbative QCD, Phys. Rev. D 68 (2003) 114012 [hep-ph/0304172] [INSPIRE].

[33] J.R. Gaunt and W.J. Stirling, Double parton distributions incorporating perturbative QCD evolution and momentum and quark number sum rules, JHEP 03 (2010) 005 [arXiv: 0910.4347] [INSPIRE].

[34] F.A. Ceccopieri, An update on the evolution of double parton distributions, Phys. Lett. B 697 (2011) 482 [arXiv:1011.6586] [INSPIRE]. 